\title{
Reverse Engineering MAC
}

\author{
Ao Tang, Jang-Won Lee, Jianwei Huang, Mung Chiang, and A. Robert Calderbank
}

\begin{abstract}
This paper reverse engineers backoff-based random-access MAC protocols in ad-hoc networks. We show that the contention resolution algorithm in such protocols is implicitly participating in a non-cooperative game. Each link attempts to maximize a selfish local utility function, whose exact shape is reverse engineered from the protocol description, through a stochastic subgradient method in which the link updates its persistence probability based on its transmission success or failure. We prove that existence of a Nash equilibrium is guaranteed in general. The minimum amount of backoff aggressiveness needed for uniqueness of Nash equilibrium and convergence of the best response strategy are established as a function of user density. Convergence properties and connection with the best response strategy are also proved for variants of the stochastic-subgradient-based dynamics of the game. Together with known results in reverse engineering TCP and BGP, this paper completes the recent efforts in reverse engineering the main protocols in layers $2-4$.
\end{abstract}

Keywords: Wireless network, Ad hoc network, Medium access control, Mathematical programming/optimization, Network utility maximization, Game theory, Network control by pricing, Reverse engineering.

\section{INTRODUCTION}

To better understand backoff-based random-access protocols in wireless MAC (Medium Access Control), such as the BEB (Binary Exponential Backoff) protocol in the IEEE 802.11 DCF standard, we pose the following question: are the distributed and selfish actions by each link in such protocols in fact implicitly maximizing some local utility functions? We answer this question by developing a non-cooperative game model for EB (Exponential Backoff) type of MAC protocols, reverse engineering the underlying utility function's form from protocol description, and establishing the existence, uniqueness, and stability properties of Nash equilibrium.

This reverse engineering effort is different from either imposing a particular utility maximization or game-theoretic model (e.g., the game-theoretic model for slotted Aloha in [12]) or performance analysis of a protocol without discovering the underlying optimization process (e.g., analysis of 802.11 protocols based on Markov model [3], [19]). While queuing-theoretic stability of contention-based MAC has been well-studied since 1970s, reverse engineering results of MAC protocols have not appeared before.

This work was supported by Yonsei University research fund of 2005 and NSF Grants CCF-0440443, CNS-0417607, CCF-0448012, and CNS-0427677.

A. Tang is with the Department of Electrical Engineering, Caltech, Pasadena, CA 91125, USA (email:aotang@caltech.edu). J.-W. Lee is with CITY - Center for Information Technology of Yonsei University, Department of Electrical and Electronic Engineering, Yonsei University, 134 Shinchondong, Seodaemun-gu, Seoul, Korea 120-749 (email: jangwon@yonsei.ac.kr). J. Huang, M. Chiang and A. R. Calderbank are with the Department of Electrical Engineering, Princeton University, Princeton, NJ 08544, USA (email: jianweih@princeton.edu, chiangm@princeton.edu, calderbk@princeton.edu).
Our layer 2 reverse engineering results complement the recent work on reverse engineering layer 4 TCP, e.g., [10], [16], and layer 3 BGP [5]. By starting with a given protocol description and working backwards to determine the underlying optimization problem implicitly solved by the protocol, much new insights on protocol performance, parameter setting, cross-layer interactions, and design improvements can be rigorously obtained, as illustrated through forward engineering successes of TCP and BGP over the last few years.

For example, Internet TCP/AQM protocols in the transport layer have recently been reverse engineered as implicitly solving a cooperative Network Utility Maximization (NUM) [7], [11], [13], [8] using different Lagrange multipliers or congestion prices. Consider a communication network with $L$ logical links, each with a fixed capacity of $c_{l}$ bps, and $S$ sources (i.e., end users), each transmitting at a source rate of $x_{s}$ bps. Each source $s$ emits one flow, using a fixed set $L(s)$ of links in its path, and has an increasing, concave utility function $U_{s}\left(x_{s}\right)$. The basic NUM problem is formulated as [7]:

$$
\begin{aligned}
& \begin{array}{ll}
\operatorname{maximize} & \sum_{s} U_{s}\left(x_{s}\right) \\
\text { subject to } & \sum_{s: l \in L(s)} x_{s} \leq c_{l}, \quad \forall l,
\end{array} \\
& \mathbf{x}^{\min } \preceq \mathbf{x} \preceq \mathbf{x}^{\max } .
\end{aligned}
$$

Even though TCP/AQM protocols were first designed without regard to global optimization, the reverse engineering model provides a rigorous path towards understanding the equilibrium and dynamic properties of complicated interactions across sources and routers and valuable guidance in design issues. In those models, the utility function of each source depends only on its data rate that can be directly controlled by the source itself, and there are adequate feedback from the network. Hence, the TCP/AQM protocol can be modeled as a distributed algorithm that converges to the globally optimal rate allocation by implicitly solving the basic NUM problem (1) for different utility functions and its Lagrange dual problem.

In contrast, in the $\mathrm{EB}$ MAC protocol, the utility of each link directly depends on not just its own transmission (e.g., persistence probability) but also transmissions of other links due to collisions that cannot be controlled by the link itself. Moreover, there is no explicit feedback from the network. Hence, a non-cooperative game model is more appropriate for the EB protocol than a global optimization model. We show that the EB protocol can be reverse engineered through a non-cooperative game in which each link tries to maximize, using a stochastic subgradient formed by local information, its own utility function in the form of expected net reward for successful transmission. While the existence of Nash equilibrium can be proved, neither convergence nor social 


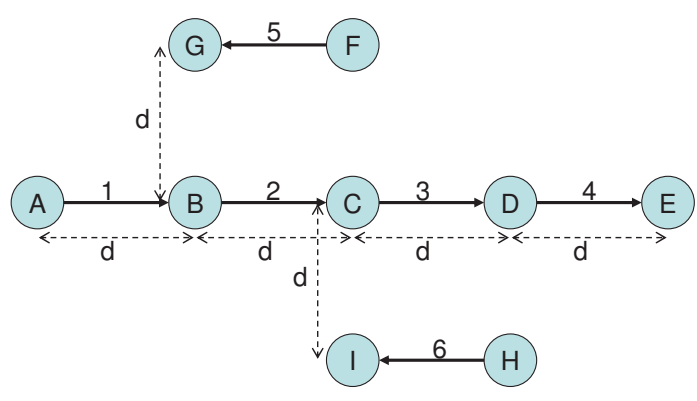

Fig. 1. Logical topology graph of an example network.

welfare optimality is guaranteed. We then provide sufficient conditions on user density and backoff aggressiveness that guarantee uniqueness and stability (i.e., convergence of the standard best response strategy) of Nash equilibrium. We also characterize a contraction region for the expected trajectory of the stochastic subgradient method, and show that a sequential variant of the stochastic subgradient method is equivalent to the best response strategy.

The rest of this paper is organized as follows. In Section II, we provide the system model. In Section III-A, we establish a non-cooperative game model for the EB protocol, reverse engineer the underlying utility function, and prove the existence of Nash equilibrium. In Section III-B, we further reverse engineer the EB protocol as a stochastic subgradient method. We characterize the uniqueness and stability properties of Nash equilibrium in Section III-C, and develop the relationship between the stochastic subgradient method and the best response strategy in Section III-D. In Section IV, we provide numerical results that illustrate the properties of the EB protocol as a non-cooperative game, and we conclude in Section V. Most of the proofs are presented in the Appendix.

\section{SySTEM MODEL}

Consider an ad-hoc network represented by a directed graph $G(V, E)$, e.g., as in Figure 1, where $V$ is the set of nodes and $E$ is the set of logical links. We define $L_{t o}^{I}(l)$ as the set of links whose transmissions cause interference to the receiver of link $l$ and $L_{\text {from }}^{I}(l)$ as the set of links whose transmissions get interfered from the transmission of link $l$. Hence, if link $l$ and a link in set $L_{t o}^{I}(l)$ transmit data simultaneously, the transmission of link $l$ fails. If link $l$ and a link $k$ in set $L_{\text {from }}^{I}(l)$ transmit data simultaneously, the transmission of link $k$ also fails.

The EB protocol is a prototypical contention resolution protocol in such wireless networks. In the IEEE 802.11 implementation, the EB protocol is window-based: each link $l$ maintains its contention window size $W_{l}$, current window size $C W_{l}$, and minimum and maximum window sizes $W_{l}^{\text {min }}$ and $W_{l}^{\max }$. After each transmission, contention window size and current window size are updated. If transmission is successful, the contention window size is reduced to the minimum window size (i.e., $W_{l}=W_{l}^{\min }$ ), otherwise it is doubled until reaching the maximum window size $W_{l}^{\max }$ (i.e., $W_{l}=\min \left\{2 W_{l}, W_{l}^{\max }\right\}$ ). Then, current window size
$C W_{l}$ is updated to be a number between $\left(0, W_{l}\right)$ following a uniform distribution. It decreases in every time-slot, and when it becomes zero, the link transmits data. Since the window size is doubled after each transmission failure, the BE protocol in the IEEE 802.11 is called the Binary Exponential Backoff (BEB) protocol, which is a special case of EB protocols.

We study the window-based EB MAC protocol through a persistence probabilistic model, an approach analogous to the source rate model in the literature for the window-based TCP congestion control protocol. Here each link $l$ transmits data with a probability $p_{l}$, which we refer to as the persistence probability of link $l$. After each transmission attempt, if the transmission is successful without collisions, then link $l$ sets its persistence probability to be its maximum value, $p_{l}^{\max }$. Otherwise, it multiplicatively reduces its persistence probability by a factor $\beta_{l}\left(0<\beta_{l}<1\right)$ until reaching its minimum value $p_{l}^{\text {min }}$.

Since in the window-based BEB protocol the current window size $C W_{l}$ of link $l$ is randomly selected between $\left(0, W_{l}\right)$, when its window size is $W_{l}$, we may think that link $l$ transmits data in a time-slot with an attempt probability $1 / W_{l}$, which corresponds to the persistence probability $p_{l}$ in our model for the average behavior of the EB protocols. In the windowbased protocol, after every transmission success, the attempt probability is set to be its maximum value (i.e., $1 / W_{l}^{\min }$ ), which corresponds to $p_{l}^{\max }$ in our model, and after every transmission failure, the attempt probability is set to be a fraction of its current value until it reaches to its minimum value, which corresponds to reducing the persistence probability by a factor of $\beta=0.5$ in BEB (and in general $\beta \in(0,1)$ in $\mathrm{EB}$ ) until reaching the minimum persistence probability $p_{l}^{\min }$.

\section{Reverse EngIneERING: NON-COOPERATIVE GAME MODEL OF EB MAC PROTOCOL}

In this section, we characterize the selfish utility maximization problem that is implicitly solved by random-access MAC protocols such as EB. In contrast to the TCP/AQM protocol that can be modeled as a basic NUM (1), we model the EB protocol as a non-cooperative game due to the coupled utility of each link through collisions and the lack of sufficient feedback from the network.

\section{A. Game Model, Utility Function, and Existence of Nash Equilibrium}

The update algorithm for the persistence probability described in the previous section can be written as:

$$
\begin{aligned}
p_{l}(t+1)= & \max \left\{p_{l}^{\min }, p_{l}^{\max } \mathbf{1}_{\left\{T_{l}(t)=1\right\}} \mathbf{1}_{\left\{C_{l}(t)=0\right\}}\right. \\
& +\beta_{l} p_{l}(t) \mathbf{1}_{\left\{T_{l}(t)=1\right\}} \mathbf{1}_{\left\{C_{l}(t)=1\right\}} \\
& \left.+p_{l}(t) \mathbf{1}_{\left\{T_{l}(t)=0\right\}}\right\},
\end{aligned}
$$

where $p_{l}(t)$ is a persistence probability of link $l$ at time-slot $t, \mathbf{1}_{a}$ is an indicator function of event $a$, and $T_{l}(t)$ and $C_{l}(t)$ are the events that link $l$ transmits data at time-slot $t$ and that there is a collision to link $l$ 's transmission given that link $l$ 
transmits data at time-slot $t$, respectively. Then, given $\mathbf{p}(t)$, we have

$$
\operatorname{Prob}\left\{T_{l}(t)=1 \mid \mathbf{p}(t)\right\}=p_{l}(t)
$$

and

$$
\operatorname{Prob}\left\{C_{l}(t)=1 \mid \mathbf{p}(t)\right\}=1-\prod_{n \in L_{t o}^{I}(l)}\left(1-p_{n}(t)\right) .
$$

Since the update of the persistence probabilities for the next time-slot depends only on the current persistence probabilities, we will consider the update conditioning on the current persistence probabilities. Note that $p_{l}(t)$ is a random process whose transitions depend on events $T_{l}(t)$ and $C_{l}(t)$. We first study its expected trajectory and will return to (2) later in this section. Slightly abusing the notation, we still use $p_{l}(t)$ to denote the expected persistence probability. From (2), we have

$$
\begin{aligned}
p_{l}(t+1)= & \max \left\{p_{l}^{\min }, p_{l}^{\max } \mathrm{E}\left\{\mathbf{1}_{\left\{T_{l}(t)=1\right\}} \mathbf{1}_{\left\{C_{l}(t)=0\right\}} \mid \mathbf{p}(t)\right\}\right. \\
& +\beta_{l} \mathrm{E}\left\{p_{l}(t) \mathbf{1}_{\left\{T_{l}(t)=1\right\}} \mathbf{1}_{\left\{C_{l}(t)=1\right\}} \mid \mathbf{p}(t)\right\} \\
& \left.+\mathrm{E}\left\{p_{l}(t) \mathbf{1}_{\left\{T_{l}(t)=0\right\}} \mid \mathbf{p}(t)\right\}\right\} \\
= & \max \left\{p_{l}^{\min }, p_{l}^{\max } p_{l}(t) \prod_{n \in L_{t o}^{I}(l)}\left(1-p_{n}(t)\right)\right. \\
& +\beta_{l} p_{l}(t) p_{l}(t)\left(1-\prod_{n \in L_{t o}^{I}(l)}\left(1-p_{n}(t)\right)\right) \\
& \left.+p_{l}(t)\left(1-p_{l}(t)\right)\right\},
\end{aligned}
$$

where $\mathrm{E}\{a \mid b\}$ is the expected value of $a$ given $b$.

We now reverse engineer the update algorithm in (3) as a game, in which each link $l$ updates its strategy, i.e., its persistence probability $p_{l}$, to maximize its utility $U_{l}$ based on strategies of the other links, i.e., $\mathbf{p}_{-l}=$ $\left(p_{1}, \cdots, p_{l-1}, p_{l+1}, \cdots, p_{|E|}\right)$.

Formally, we formulate the EB protocol as a noncooperative game, $G_{E B-M A C}=\left[E, \times_{l \in E} A_{l},\left\{U_{l}\right\}_{l \in E}\right]$, where $E$ is a set of players, i.e., links, $A_{l}=\left\{p_{l} \mid p_{l}^{\text {min }} \leq p_{l} \leq\right.$ $\left.p_{l}^{\max }\right\}$ is an action set of player $l$, and $U_{l}$ is a utility function of player $l$ to be determined through reverse engineering. We refer to this as the EB-MAC Game and now study its properties and solutions.

In the non-cooperative game, some of the most important questions are the existence, uniqueness, and stability of its Nash equilibrium [15]. In the case of the EB-MAC Game, we have the following definition of Nash equilibrium.

Definition 1: A persistence probability vector $\mathbf{p}^{*}$ is said to be a Nash equilibrium if no link can improve its utility by unilaterally deviating its persistence probability from Nash equilibrium:

$$
U_{l}\left(p_{l}^{*}, \mathbf{p}_{-l}^{*}\right) \geq U_{l}\left(p_{l}, \mathbf{p}_{-l}^{*}\right), p_{l}^{\text {min }} \leq p_{l} \leq p_{l}^{\max }, \quad \forall l .
$$

The following reverse engineering theorem, proved in Appendix VI-A, obtains the underlying utility functions in the EB-MAC Game and establishes the existence of Nash equilibrium for the game.

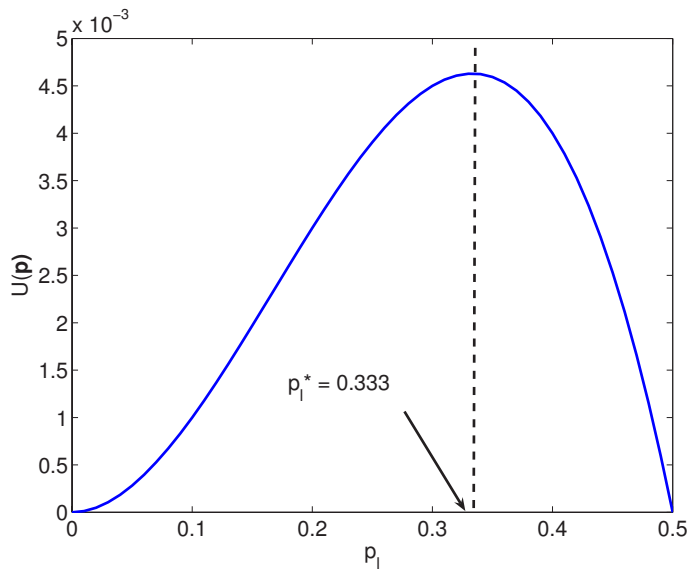

Fig. 2. Dependence of a utility function on its own persistence probability, for $\beta_{l}=0.5, p_{l}^{\max }=0.5$, and $\prod_{n \in L_{t o}^{I}(l)}\left(1-p_{n}\right)=0.5$.

Theorem 1: The utility function is the following expected net reward (expected reward minus expected cost) that the link can obtain from its transmission:

$$
U_{l}(\mathbf{p})=R\left(p_{l}\right) S(\mathbf{p})-C\left(p_{l}\right) F(\mathbf{p}), \quad \forall l
$$

where $S(\mathbf{p})=p_{l} \prod_{n \in L_{t o}^{I}(l)}\left(1-p_{n}\right)$ is the probability of transmission success, $F(\mathbf{p})=p_{l}\left(1-\prod_{n \in L_{t o}^{I}(l)}\left(1-p_{n}\right)\right)$ is the probability of transmission failure, and $R\left(p_{l}\right) \stackrel{\text { def }}{=} p_{l}\left(\frac{1}{2} p_{l}^{\max }-\right.$ $\left.\frac{1}{3} p_{l}\right)$ can be interpreted as the reward for transmission success, $C\left(p_{l}\right) \stackrel{\text { def }}{=} \frac{1}{3}\left(1-\beta_{l}\right) p_{l}^{2}$ can be interpreted as the cost for transmission failure.

Furthermore, there exists a Nash equilibrium in the EBMAC Game $G_{E B-M A C}=\left[E, \times_{l \in E} A_{l},\left\{U_{l}\right\}_{l \in E}\right]$ characterized by the following:

$$
p_{l}^{*}=\frac{p_{l}^{\max } \prod_{n \in L_{t o}^{I}(l)}\left(1-p_{n}^{*}\right)}{1-\beta_{l}\left(1-\prod_{n \in L_{t o}^{I}(l)}\left(1-p_{n}^{*}\right)\right)}, \forall l .
$$

Remark: It is important to note that the expressions of $S(\mathbf{p})$ and $F(\mathbf{p})$ come directly from the definitions of success and failure probabilities, while the expressions of $R\left(p_{l}\right)$ and $C\left(p_{l}\right)$ (thus exact form of $U_{l}$ ) are in fact derived in the proof by reverse engineering the EB protocol description. Both $R\left(p_{l}\right)$ and $C\left(p_{l}\right)$ turn out to be quadratic functions of $p_{l}$.

From (5), we conclude that, other conditions being the same, at a Nash Equilibrium a link $l$ will have a higher persistence probability if it has a higher value of $p_{l}^{\max }$, a higher value of $\beta_{l}$, or a higher value of $\prod_{n \in L_{+o}^{I}(l)}\left(1-p_{n}^{*}\right)$, i.e., a higher transmission success probability. We also have the next corollaries that immediately follow from (3) and (5).

Corollary 1: If $\mathbf{p}(t)$ updated by (3) converges to $\mathbf{p}^{*}$, $\mathbf{p}^{\min }<\mathbf{p}^{*}<\mathbf{p}^{\max }$, then $\mathbf{p}^{*}$ is a Nash equilibrium.

Corollary 2: Suppose that $p_{l}^{\text {min }}>0, \forall l, p_{l}^{*} \rightarrow$ $p_{l}^{\text {min }}$ as $\left|L_{t o}^{I}(l)\right| \rightarrow \infty$.

Corollary 3: Suppose that $p_{l}^{\text {min }}=0, \forall l$. Let $\left|L_{t o}^{I}(l)\right| \rightarrow$ $\infty$. If $p_{l}^{*}>0$, then only a finite number of links among links in $L_{t o}^{I}(l)$ have positive persistence probabilities at a Nash equilibrium. 
Corollaries 2 and 3 can be easily proven with (17) in Appendix VI-A and the fact that, as the number of links in $L_{t_{o}}^{I}(l)$ with a positive persistence probability at a Nash equilibrium goes to infinity, $p_{l}^{*}$ in (5) goes to zero. Corollaries 2 and 3 confirm the intuition that, as the number of interfering nodes to a link increases (i.e., as the amount of contention in the contention region of a link gets higher), the persistence probability of the link decreases.

\section{B. EB Protocol and Stochastic Subgradient Method}

Using (15), we can rewrite (3) as

$$
p_{l}(t+1)=\max \left\{p_{l}^{\text {min }}, p_{l}(t)+\left.\frac{\partial U_{l}(\mathbf{p})}{\partial p_{l}}\right|_{\mathbf{p}=\mathbf{p}(t)}\right\} \text {. }
$$

Hence, in (3), each link updates its persistence probability to the direction of the maximizer using the gradient. To update its persistence probability by (3), each link $l$ must know the persistence probabilities of its adjacent links, i.e., link $n$, $n \in L_{t o}^{I}(l)$. However, in the EB protocol, there is no explicit message passing among links, and the link cannot obtain the exact information to evaluate the gradient of its utility function. Instead of using the exact gradient of its utility function as in (3), each link attempts to approximate it using (2). In fact, we can rewrite (2) as

$$
\begin{aligned}
p_{l}(t+1)= & \max \left\{p_{l}^{\min }, p_{l}(t)-p_{l}(t)+p_{l}^{\max } \mathbf{1}_{\left\{T_{l}(t)=1\right\}} \mathbf{1}_{\left\{C_{l}(t)=0\right\}}\right. \\
& \left.+\beta_{l} p_{l}(t) \mathbf{1}_{\left\{T_{l}(t)=1\right\}} \mathbf{1}_{\left\{C_{l}(t)=1\right\}}+p_{l}(t) \mathbf{1}_{\left\{T_{l}(t)=0\right\}}\right\} \\
= & \max \left\{p_{l}^{\text {min }}, p_{l}(t)+v_{l}(t)\right\},
\end{aligned}
$$

where

$$
\begin{aligned}
v_{l}(t)= & p_{l}^{\max } \mathbf{1}_{\left\{T_{l}(t)=1\right\}} \mathbf{1}_{\left\{C_{l}(t)=0\right\}} \\
& +\beta_{l} p_{l}(t) \mathbf{1}_{\left\{T_{l}(t)=1\right\}} \mathbf{1}_{\left\{C_{l}(t)=1\right\}} \\
& +p_{l}(t) \mathbf{1}_{\left\{T_{l}(t)=0\right\}}-p_{l}(t) .
\end{aligned}
$$

Since

$$
\begin{aligned}
\mathrm{E}\left\{v_{l}(t) \mid \mathbf{p}(t)\right\}= & p_{l}^{\max } p_{l}(t) \prod_{n \in L_{t o}^{I}(l)}\left(1-p_{n}(t)\right) \\
& +\beta_{l} p_{l}(t) p_{l}(t)\left(1-\prod_{n \in L_{t o}^{I}(l)}\left(1-p_{n}(t)\right)\right) \\
& +p_{l}(t)\left(1-p_{l}(t)\right)-p_{l}(t) \\
= & \left.\frac{\partial U_{l}(\mathbf{p})}{\partial p_{l}}\right|_{\mathbf{p}=\mathbf{p}(t)},
\end{aligned}
$$

we conclude that $v_{l}(t)$ is a stochastic subgradient [6] of $U_{l}$ at $\mathbf{p}(t)$.

In summary, we have the following reverse engineering result in addition to Theorem 1:

Theorem 2: The EB protocol described by (2) is a stochastic subgradient algorithm to maximize utility (4).

Remark: Each stochastic subgradient $v_{l}$ can be measured by the link itself through collision and success of its transmission, without explicit message passing among links.

We now examine dynamics of (3) with a tunable step size $\gamma$ (the actual protocol is implicitly using a constant step size of 1):

$$
p_{l}(t+1)=\max \left\{p_{l}^{\text {min }}, p_{l}(t)+\left.\gamma \frac{\partial U_{l}(\mathbf{p})}{\partial p_{l}}\right|_{\mathbf{p}=\mathbf{p}(t)}\right\} .
$$

We start with the following definition:

Definition 2: A set $R_{l}$ is called a contraction region for a dynamical system $\mathbf{p}(t+1)=f(\mathbf{p}(t))$ if there exists an integer $N$ such that for all initial conditions, $p_{l}(n) \in R_{l}$ for some $n$ less than $N$.

Although after the trajectory enters the contraction region it may still leave, the definition guarantees that it will come back within $N$ steps. When strict convergence result is not available, a contraction region characterization describes the main region where the system stays.

Now consider the aggregate utility $V=\sum_{l} U_{l}(\mathbf{p})$. It can be shown that $\nabla V$ is Lipschitz continuous with a Lipschitz constant that we denote as $C$. If $\mathbf{p}(t+1)=\mathbf{p}(t)+\gamma \nabla V$, then clearly the system converges with proper step-size $\gamma<\frac{2}{C}$. Due to the lack of knowledge of other links' persistence probability and the non-cooperative nature of the game, the actual update direction is $\frac{\partial U_{l}(\mathbf{p})}{\partial p_{l}}$ instead of the gradient $\nabla V$. Consider the difference between these two vectors:

$$
\frac{\partial U_{l}(\mathbf{p})}{\partial p_{l}}-\frac{\partial V}{\partial p_{l}}=-\sum_{n \in L_{t o}^{I}(l)} \frac{\partial U_{n}(\mathbf{p})}{\partial p_{l}}, \forall l .
$$

The following inequality characterizes $\mathbf{p}$ for which the error is not large enough to negate the exact gradient and $V$ still increases after each step:

$$
-\sum_{n \in L_{t o}^{I}(l)} \frac{\partial U_{n}(\mathbf{p})}{\partial p_{l}}<\mu \frac{\partial U_{l}(\mathbf{p})}{\partial p_{l}}
$$

where $\mu \in[0,1)$ can be viewed as the relative error.

When (8) fails to hold, the following opposite inequality holds, and leads to a contraction region characterization:

$$
-\sum_{n \in L_{t o}^{I}(l)} \frac{\partial U_{n}(\mathbf{p})}{\partial p_{l}} \geq \mu \frac{\partial U_{l}(\mathbf{p})}{\partial p_{l}} .
$$

Theorem 3: The solution of (9) is an contraction region for (6) with step-size $\gamma \in(0,2 / C(1-\mu))$

The basic idea of the proof is that once $\mathbf{p}$ is out of the region defined by (9), then (8) holds and $V$ increases after each step. As $V$ is finite and the increments are lower-bounded, after a finite number of steps, (8) will fail and the system will get into the contraction region again. The detailed proof is omitted here as it consists of a standard argument using the Descent Lemma [2], as was done in [14] for a different problem.

\section{Uniqueness of Nash Equilibrium and Convergence of Best Response}

In Theorem 1, we have shown that there exists a Nash equilibrium in the EB-MAC game. However, in general, there may not be a unique Nash equilibrium, as illustrated in a simple example. Suppose that there are two links interfering with each other, and that $p_{1}^{\max }=p_{2}^{\max }=p^{\max }=1$, then 
it can be verified that there is an infinite number of Nash equilibria, which is the set of $\left(p_{1}^{*}, p_{2}^{*}\right)$ satisfying

$$
\max \left\{p^{\min }, \frac{1-p^{\max }}{1-\beta p^{\max }}\right\} \leq p_{1}^{*} \leq \min \left\{1, \frac{1-p^{\min }}{1-\beta p^{\min }}\right\}
$$

and

$$
p_{2}^{*}=\frac{1-p_{1}^{*}}{1-\beta p_{1}^{*}} .
$$

We will investigate uniqueness of Nash equilibrium together with the convergence of a natural strategy for the game: the best response strategy, commonly used to study stability of Nash equilibrium. In best response, each link updates its persistence probability for the next time-slot such that it maximizes its utility based on the persistence probabilities of the other links in the current time-slot:

$$
p_{l}^{*}(t+1)=\underset{p_{l}^{\text {min }} \leq p_{l} \leq p_{l}^{\max }}{\operatorname{argmax}} U_{l}\left(p_{l}, \mathbf{p}_{-l}^{*}(t)\right) .
$$

Hence, $p_{l}^{*}(t+1)$ is the best response of link $l$ given $\mathbf{p}_{-l}^{*}(t)$.

Note that, in current practice, the persistence probability in the EB protocol is not updated by the best response strategy, but by (2) (or by (3) on average). Hence, in the EB protocol, instead of instantaneously setting $p_{l}(t+1)$ to the best response $p_{l}^{*}(t+1)$, in (2) (or (3)) each link updates its persistence probability to the direction of the maximizer by using the stochastic gradient. Hence, in the EB protocol, the persistence probability of the link is updated more smoothly than the best response.

Based on S-modular game theory as shown in Appendix VI$\mathrm{B}$, the following theorem provides our first characterization of the convergence properties of the best response strategy to a Nash equilibrium in the EB-MAC Game.

Theorem 4: Suppose that the persistence probability of each link is updated by the best response function in (10) in each time-slot with $\mathbf{p}^{*}(0)=\mathbf{p}^{\text {min }}$. Then,

$$
\mathbf{p}^{*}(2 t+1) \rightarrow \hat{\mathbf{p}} \text { and } \mathbf{p}^{*}(2 t) \rightarrow \tilde{\mathbf{p}} \text { as } t \rightarrow \infty
$$

If $\hat{\mathbf{p}}=\tilde{\mathbf{p}}$ i.e., if $\mathbf{p}^{*}(t)$ converges to $\hat{\mathbf{p}}$, then $\hat{\mathbf{p}}$ is a Nash equilibrium.

Thus far, we have shown that Nash equilibrium of the EBMAC game may not be unique and, further, the best response strategy may not converge to a Nash equilibrium. However, by imposing some conditions on the strategy set of each link, we can guarantee both the uniqueness of Nash equilibrium and the convergence of the best response strategy to the Nash equilibrium.

For notational simplicity, we assume all links have the same $p^{\max }$ and $p^{\min }$. Furthermore, assume that $p^{\max }<1$ and $p^{\text {min }}=0^{1}$. Then, from (5), we have

$$
p_{l}^{*}=p^{\max } \frac{\prod_{n \in L_{t o}^{I}(l)}\left(1-p_{n}^{*}\right)}{1-\beta\left(1-\prod_{n \in L_{t o}^{I}(l)}\left(1-p_{n}^{*}\right)\right)},
$$

${ }^{1}$ If the maximum window size is sufficient large, then $p^{\text {min }}$ can be sufficient close to 0 . And if we don't allow the minimum window to be 1 , which is a plausible thing to do, then the smallest minimum window is 2 and the corresponding $p^{\max }=0.5<1$. where $L_{t o}^{I}(l)$ is a set of links that cause interference to link $l$. We first bound Nash equilibrium with the following

Lemma 1: We have $p_{l}^{*}>0$ and $p_{l}^{*}<p^{\max }$.

This lemma is proved in Appendix VI-C and guarantees that any equilibrium must be an inner solution. We now show that when contention density is not too high, the above solution is actually the unique Nash equilibrium.

Let $K=\max _{l}\left\{\left|L_{t o}^{I}(l)\right|\right\}$, which captures the amount of potential contention among links. We have the following theorem that relates three key quantities: amount of potential contention $K$, backoff multiplier $\beta$ (speed of backoff), and $p^{\max }$ that corresponds to the minimum contention window size (minimum amount of backoff).

Theorem 5: If $\frac{p^{\max } K}{4 \beta\left(1-p^{\max }\right)}<1$, then

1) The Nash equilibrium is unique;

2) Start from any initial point, the iteration defined by best response converges to the unique equilibrium.

The proof is in Appendix VI-D. The key idea is to show the updating rule from $p(t)$ to $p(t+1)$ is a contraction mapping [1] by verifying a particular norm of the Jacobian $\mathbf{J}$ of the update dynamics in the game $\left(\|\mathbf{J}\|_{\infty}\right.$ in our proof) is less than one.

There are several interesting engineering implications from the above theorem. For example, it provides a guidance to choose parameter in the EB protocols, and quantifies the intuition that with a large enough $\beta$ (i.e., links do not decrease the probabilities suddenly) and a small enough $p^{\max }$ (i.e., links backoff aggressively enough), uniqueness and stability can be ensured. The higher the amount of contention (i.e., a larger value of $K$ ), the smaller $p^{\max }$ needs to be.

Some of the other implications are stated in the following corollary, whose proof hinges upon the following observation. If $\beta \leq 0.5$, then $\frac{1-\beta}{\beta(1-p)} \geq 1$ for $p \in(0,1)$, and we have

$$
\begin{aligned}
\|\mathbf{J}\|_{\infty} & \leq \max _{l}\left\{\frac{p^{\max }\left|L_{t o}^{I}(l)\right|(1-\beta)}{\left(1-\beta+\beta\left(1-p^{\max }\right)\right)^{2}}\right\} \\
& \leq \frac{p^{\max } K(1-\beta)}{\left(1-\beta+\beta\left(1-p^{\max }\right)\right)^{2}} .
\end{aligned}
$$

Corollary 4: If any one of the following conditions is satisfied, then the Nash equilibrium is unique. Moreover, starting from any initial point, the iteration defined by best response converges to the unique equilibrium.

$$
\beta \leq 0.5 \text { and } \frac{p^{\max } K(1-\beta)}{\left(1-\beta+\beta\left(1-p^{\max }\right)\right)^{2}}<1 ;
$$

(b) For the system in which each link interferes each other (i.e., $\left.L_{t o}^{I}(l)=E-\{l\}, \forall l\right)$, e.g., as in an uplink topology, $\frac{p^{\text {nax }}(L-1)}{4 \beta\left(1-p^{\text {max }}\right)}<1$, where $L$ is the number of links;

(c) For the system in which each link interferes each other (i.e., $\left.L_{t o}^{I}(l)=E-\{l\}, \forall l\right), \beta \leq 0.5$ and $\frac{p^{\max }(L-1)(1-\beta)}{\left(1-\beta+\beta\left(1-p^{\max }\right)\right)^{2}}<1$.

Remark: Part (c) of the above corollary quantifies the intuition that smaller number of interfering links helps uniqueness and stability of Nash equilibrium: $L$ needs to be smaller than $1+\frac{\left(1-\beta+\beta\left(1-p^{\max }\right)\right)^{2}}{p^{\max }(1-\beta)}$.

Interpreting the above results in another way, we examine the dependence of the maximum $p^{\max }$ allowed, i.e., the least 


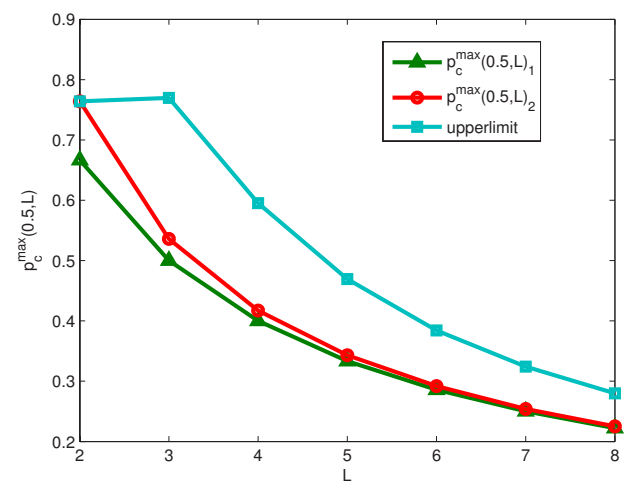

Fig. 3. $p_{c}^{\max }(\beta, L)$ and its upperlimit for $\beta=0.5$.

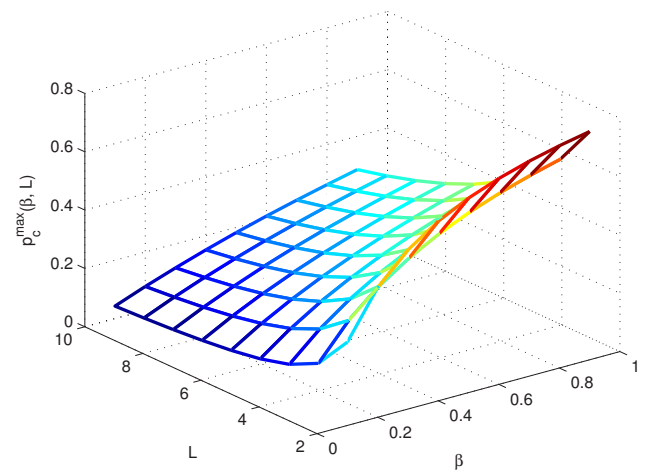

Fig. 4. $p_{c}^{\max }(\beta, L)$ from Corollary $4(\mathrm{~b})$.

amount of backoff needed in terms of the smallest $W^{\text {min }}$, in order to ensure uniqueness and stability of EB protocol, as a function of backoff multiplier $\beta$ and user density $L$. Using $p_{c}^{\max }(\beta, L)$ to denote the critical value of $p^{\max }$ satisfying the bounds, both $p_{c}^{\max }(\beta, L)$ developed in Corollaries 4 (b) $\left(p_{c}^{\max }(\beta, L)_{1}\right)$ and 4 (c) $\left(p_{c}^{\max }(\beta, L)_{2}\right)$ are visualized in Figure 3 with the standard parameter $\beta=0.5$. It is worthwhile to note that as long as the minimum window size is 5 or larger, then for the number of active links $L$ up to 8 , which is a reasonably large number in many applications, uniqueness and convergence can be guaranteed (because $p_{c}^{\max }$ is slightly larger than $1 / 5=0.2$ when $L=8$ ).

We also plot $p_{c}^{\max }(\beta, L)$ for Corollary 4 (b) in Figure 4. Not surprisingly, $p_{c}^{\max }(\beta, L)$ in an increasing function on $\beta$ and decreasing on $L$. Moreover, it is concave on $\beta$ and convex on $L$.

A natural question to ask next is whether the above upperbounds on $p_{l}^{\max }$ are too conservative due to relaxations during the computation of bounds on Jacobian's infinity norm. The answer is no, for the contraction mapping technique used above. An upperlimit that sets the best possible upperbound we can achieve via contraction mapping using infinity norm is derived by finding the lowerbound of the maximum of $\|\mathbf{J}\|_{\infty}$, see Appendix VI-E for details. The upperlimit is also illustrated in Figure 3 with $\beta=0.5$. It is clear that the upperlimit has qualitatively the same shape as the bounds in
Corollary 4.

D. Relating Stochastic Subgradient Method with Best Response Strategy

We have shown that the stochastic subgradient updates (2) is how EB protocol works, and characterized a contraction region for its expected trajectory. A different update rule, the best response strategy (10), is the standard game-theoretic dynamics whose convergence characterizes the stability of Nash equilibrium, and we have provided sufficient conditions for its convergence. In this subsection, we develop the connection between these two updates.

Consider the case where only link $l$ updates its persistent probability $p_{l}$ similar to (2) but with a diminishing step-size, and other links contend for the common channel with fixed probabilities $\mathbf{p}_{-l}$. We can show that such sequential stochastic subgradient updates converge to the best response solution in (10) under properly chosen step-size and mild conditions of the system parameters.

Formally, define the new update algorithm for link $l$ under fixed value of $\mathbf{p}_{-l}$ as:

$$
p_{l}(t+1)=\max \left\{\tilde{p}_{l}^{\min }, \min \left\{p_{l}^{\max }, p_{l}(t)+\alpha_{l}(t) v_{l}(t)\right\}\right\},
$$

where $v_{l}(t)$ is the stochastic subgradient defined in (6), $\alpha_{l}(t)$ is the step-size, and $\tilde{p}_{l}^{\min }$ is the modified minimum persistent probability. Assume for simplicity that all users have the same minimum and maximum persistent probabilities $0 \leq p^{\min } \leq$ $p^{\max }<1$, and a common backoff multiplier $\beta$. The following result is proved in Appendix VI-F.

Theorem 6: The updates in (13) converge to the best response solution of user $l$ in (10) under fixed $\mathbf{p}_{-l}$ with probability 1 if the following conditions all hold:

1) The step-size $\alpha(t)$ satisfies $\alpha(t) \geq 0, \sum_{t=0}^{\infty} \alpha(t)=$ $\infty, \sum_{t=0}^{\infty} \alpha^{2}(t)<\infty$, e.g., $\alpha(t)=1 / t$.

2) The modified minimum persistent probability $\tilde{p}_{l}^{\min }=$ $\frac{p^{\max }\left(1-p^{\min }\right)^{M_{l}}}{1-\beta\left(1-\left(1-p^{\min }\right)^{M_{l}}\right)} \geq p^{\min }$.

3) The values of $p^{\min }, p^{\max }$ and $\beta$ satisfy $\frac{1-\beta}{\beta}\left(\frac{1}{\left(1-p^{\max }\right)^{M_{l}}}-\frac{2}{\left(1-p^{\min }\right)^{M_{l}}}\right) \leq 1, \quad$ where $M_{l}=\left|L_{t o}^{I}(l)\right|$ is the number of interfering links with link $l$.

Remark: Theorem 6 shows that although link $l$ neither knows the exact values of other links' persistent probabilities, nor has memory of other links' past behaviors, the stochastic subgradient updates can still converge to the best response strategy, if it is sequential and use diminishing step-sizes (condition 1 above).

We now show that conditions 2 and 3 in Theorem 6 are very mild and almost always satisfied in practice. Both are on system parameters: the upperbound constraint on $p^{\min }$ in condition 2, and the relationship in condition 3 . If $p^{\min }=$ 0 as assumed in Section III-C, then condition 2 always holds, and a sufficient condition for condition 3 to hold is $2\left(1-p^{\max }\right)^{M_{l}} \geq 1$. 
To see how often conditions 2 and 3 hold in practice, consider the system parameters specified in 802.11 standard (e.g., [3]). For an infrared (IR) physical layer, the minimum and maximum contention window sizes are $W_{l}^{\mathrm{min}}=64$ and $W_{l}^{\max }=1024$, which correspond to $p^{\min }=1 / 1024$ and $p^{\max }=1 / 64$ in our probabilistic model. In Figure 5, we plot the minimum value of $\beta$ that satisfies condition 3 as a function of the number of interfering links $M_{l}$. It is clear from the figure that any nonnegative value of $\beta$ satisfies condition 3 when $M_{l} \leq 45$. For any $\beta \geq 0.5$, condition 3 is satisfied with $M_{l} \leq 72$, which is large enough even for a dense network. For other physical layer specifications such as Frequency Hopping Spread Spectrum (FHSS) and Direct Sequence Spread Spectrum (DSSS), the minimum contention window sizes are $W_{l}^{\min }=16$ and $W_{l}^{\text {min }}=32$, respectively [3]. The maximum contention window sizes are the same as in the IR case. As a result, any $\beta \geq 0.5$ satisfies condition 3 when $M_{l} \leq 17$ and $M_{l} \leq 35$, for FHSS and DSSS respectively. For all three physical layer specifications, condition 2 is satisfied for any values of $\beta$ and $M_{l}$.

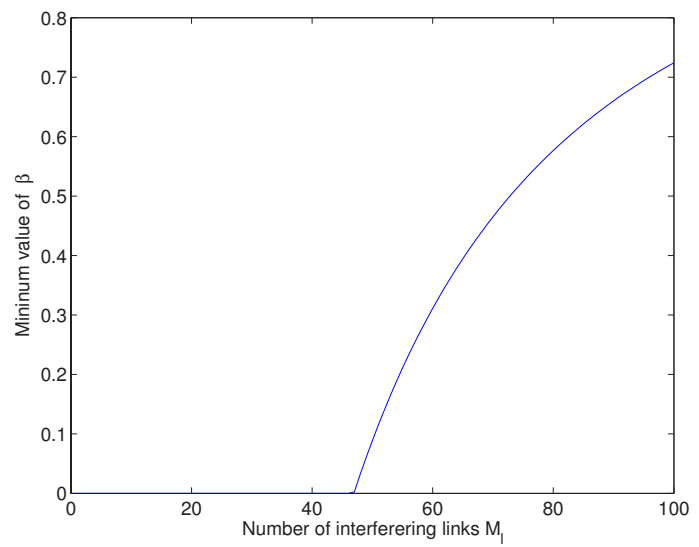

Fig. 5. The minimum value of $\beta$ that satisfies condition 3 of Theorem 6 vs. the number of interfering links $M_{l}$, for the infrared physical layer model in 802.11 [3]

\section{NUMERICAL EXAMPLES}

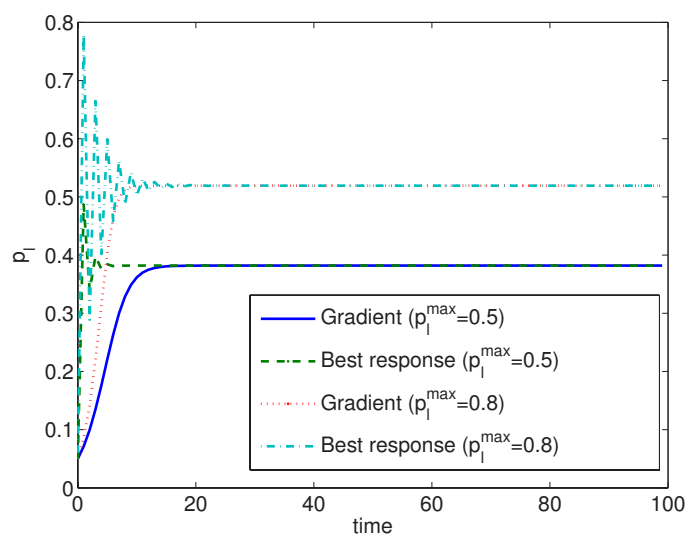

Fig. 6. Comparison of trajectories of $p_{l}(t)$ in a system with two links.

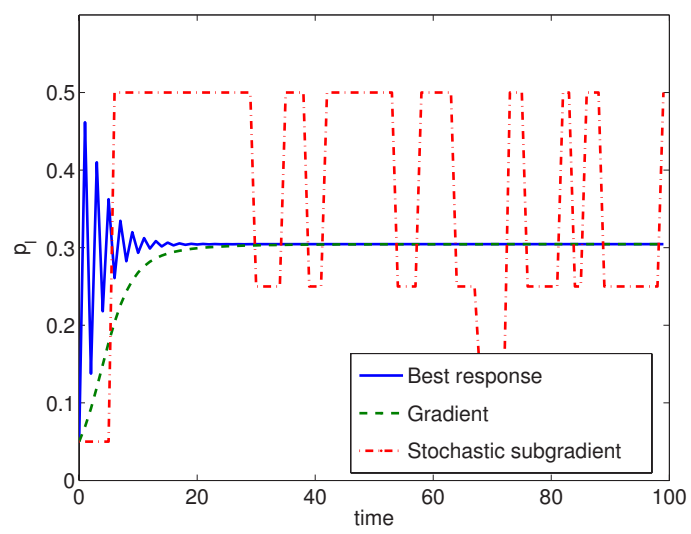

Fig. 7. Comparison of trajectories of $p_{l}(t)$ in the network in Figure 1, with $p_{l}^{\max }=0.5$.

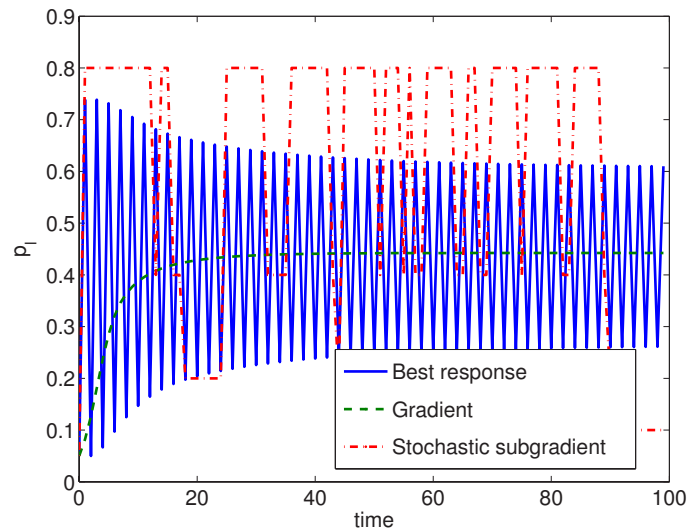

Fig. 8. Comparison of trajectories of $p_{l}(t)$ in the network in Figure 1, with $p_{l}^{\max }=0.8$.

We present numerical results for our non-cooperative game model for MAC protocol. In Figure 6, we consider a network with two links. We provide the results with $p_{l}^{\max }=0.5$ and $p_{l}^{\max }=0.8$ in the same graph, setting $\beta_{l}=0.5$ and $p_{l}^{\text {min }}=$ 0.05 for both cases. We compare trajectories of the persistence probability of link $1, p_{1}(t)$, which are obtained by (3), i.e., by gradient updates, and by (10), i.e., by best response. It can in fact be proved that, in the two-link case, the trajectory of the persistence probability obtained by (10) converges to a Nash equilibrium, which is confirmed in this numerical example. The trajectory obtained by (3) converges to the same Nash equilibrium, but more smoothly than that obtained by (10).

In Figures 7 and 8, we consider the network in Figure 1 , which has six logical links, with $\beta_{l}=0.5$ and $p_{l}^{\text {min }}=$ 0.05. In these figures, we also provide trajectories obtained by (2), i.e., by stochastic subgradient. ${ }^{2}$ In Figure 7 , we set $p_{l}^{\max }=0.5$. The figure shows that trajectories obtained by (3) and (10) converge to the same equilibrium, which must be a Nash equilibrium from Theorem $4 .{ }^{3}$ In Figure 8 , we set

\footnotetext{
${ }^{2}$ Since $p_{l}(t)$ is a stochastic process in this case, we plot its sample path.

${ }^{3}$ Although not shown in the graph, trajectories of the persistence probabilities of the other links also converge.
} 


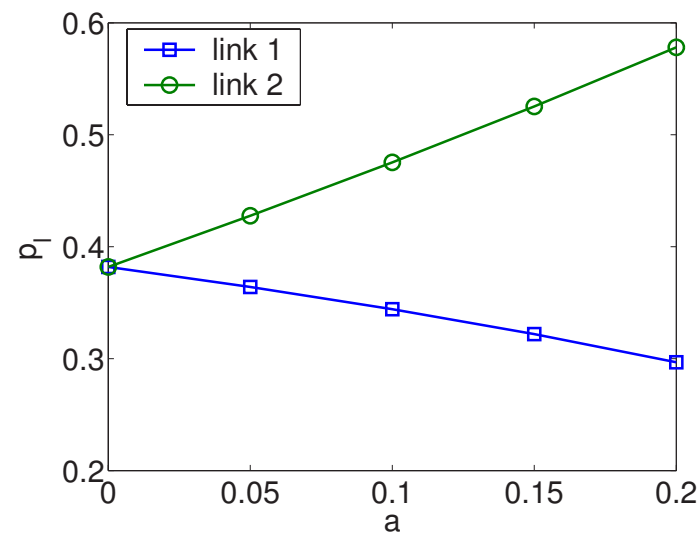

Fig. 9. Comparison of the persistence probability of links that have different $p_{l}^{\max }: \beta_{1}=\beta_{2}=0.5$, but $p_{1}^{\max }=0.5$ and $p_{2}^{\max }=0.5+a$.

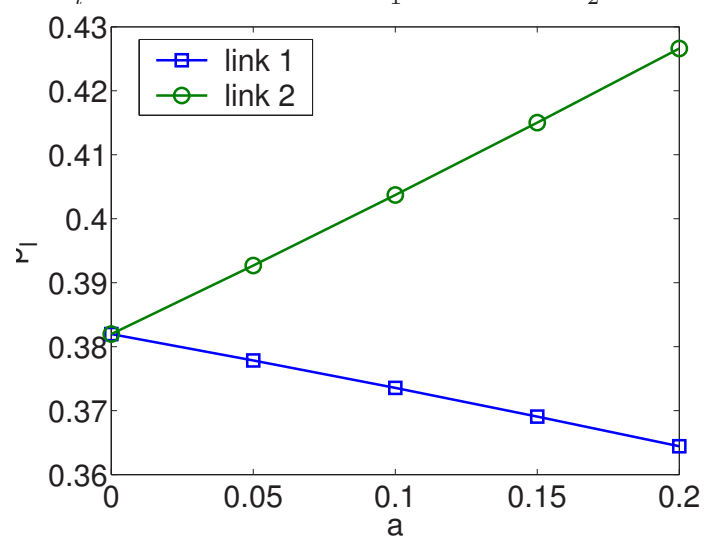

Fig. 10. Comparison of the persistence probability of links that have different $\beta_{l}: p_{1}^{\max }=p_{2}^{\max }=0.5$, but $\beta_{1}=0.5$ and $\beta_{2}=0.5+a$.

$p_{l}^{\max }=0.8$. The figure shows that the trajectory obtained by (10) oscillates between two values. Indeed, as shown in Theorem 4, in general the EB-MAC Game with the best response strategy may not converge to a Nash equilibrium. Furthermore, while the trajectory obtained by gradient method (3) converges and, by Corollary 1 , it indeed converges to a Nash equilibrium, the stochastic subgradient iterations do not converge in this example. In other simulations, we observe that the moving average of the stochastic subgradient updates with a diminishing step-size converges.

In Figures 9 and 10, we consider a two-link topology and compare the attained Nash equilibrium when each link has a different $p_{l}^{\max }$ and a different $\beta_{l}$, respectively. In Figure 9, we set $\beta_{1}=\beta_{2}=0.5$. But link 1 has its maximum persistence probability $p_{1}^{\max }=0.5$ and link 2 has its maximum persistence probability $p_{2}^{\max }=0.5+a$. In Figure 10 , we set $p_{1}^{\max }=p_{2}^{\max }=0.5$. But link 1 has $\beta_{1}=0.5$ and link 2 has $\beta_{2}=0.5+a$. Hence, in both figures, as the value of $a$ gets larger, link 2 updates its persistence probability more aggressively than link 1 . As a consequence, link 2 converges to a higher persistence probability and link 1 to a lower persistence probability, with the difference between the two increasing as the value of $a$ becomes larger. This implies that parameter setting of a link affects not only the performance of the corresponding link but also the performance of other links, causing fairness issues at the Nash equilibrium.

\section{CONCLUSions}

Starting with given protocol specifications, we have reverse engineered exponential-backoff MAC protocols as a noncooperative game where each link is implicitly maximizing, through a stochastic subgradient update, a quasi-concave utility function in the form of net reward for successful transmission. Due to the lack of proper feedback mechanisms in the current EB protocols, such selfish, local actions are not aligned to maximize the network-wide total utility, nor are they guaranteed to converge even though a Nash equilibrium for the MAC game always exists. We have also characterized a contraction region for the stochastic subgradient method, provided sufficient conditions (on user density and backoff aggressiveness) that guarantee both the uniqueness of Nash equilibrium and convergence of the best response strategy, and developed the connection between stochastic subgradient and best response for the EB-MAC game.

Our MAC layer reverse engineering results, together with the recently established reverse engineering optimization models for TCP and BGP, provide an utility-optimization-based foundation for layers 2-4 protocols. Deficiencies of existing MAC protocols revealed through reverse engineering also motivates forward engineering, where adequate feedback is generated to align selfish utility maximization by each logical link to maximize the social welfare [9].

The formulation and results in this paper can be a basis to further study other properties of EB MAC protocols, such as efficiency loss of the non-cooperative game compared with social welfare maximization. Like the reverse engineering models of TCP and BGP, there are several simplifying assumptions in our model, notably our focus only on the contention resolution mechanism. A next step is to reverse engineer carrier-sensing-based (e.g., RTS-CTS) MAC protocols (e.g., CSMA/CA) that consists of both contention avoidance and collision resolution algorithms. Finally, session level stochastic effects need to be incorporated to include the arrival statistics of finite-duration sessions. Then MAC protocols can be analyzed and designed using both stochastic stability results in traditional queuing models and optimality results in the utility maximization models.

\section{REFERENCES}

[1] R. Abraham, J. Marsden, and T. Ratiu, Manifolds, Tensor Analysis, and Applications. Springer-Verlag, 1988.

[2] D. Bertsekas, Nonlinear Programming. Athena Scientific, 1995.

[3] G. Bianchi, "Performance analysis of the IEEE 802.11 distributed coordination function," IEEE J. Sel. Area Comm., vol. 18, no. 3, pp. 535-547, March 2000.

[4] Y. Ermoliev and R. J.-B. Wets, Eds., Numerical Techniques for Stochastic Optimization. Springer-Verlag, 1980.

[5] T. G. Griffin, F. B. Shepherd, and G. Wilfong, "The stable path problem and interdomain routing," IEEE/ACM Trans. on Networking, vol. 10, no. 2, pp. 232-243, April 2002.

[6] P. Kall and S. W. Wallace, Stochastic Programming. Wiley, 1994. 
[7] F. P. Kelly, A. K. Maulloo, and D. K. H. Tan, "Rate control in communication networks: shadow prices, proportional fairness and stability," Journal of the Operational Research Society, vol. 49, no. 3, pp. 237-252, March 1998.

[8] R. J. La and V. Anantharam, "Utility-based rate control in the Internet for elastic traffic," IEEE/ACM Trans. on Networking, vol. 10, no. 2, pp. 272-286, April 2002.

[9] J. W. Lee, M. Chiang, and A. R. Calderbank, "Utility-optimal medium access control: Reverse and forward engineering," Proc. IEEE INFOCOM, April 2006.

[10] S. H. Low, "A duality model of TCP and queue management algorithms," IEEE/ACM Trans. on Networking, vol. 11, no. 4, pp. 525-536, August 2003.

[11] S. H. Low and D. E. Lapsley, "Optimization flow control-I: basic algorithm and convergence," IEEE/ACM Trans. on Networking, vol. 7, no. 6, pp. 861-874, December 1999.

[12] A. B. MacKenzie and S. B. Wicker, "Stability of multipacket slotted Aloha with selfish users and perfect information," Proc. IEEE INFOCOM, April 2003.

[13] J. Mo and J. Walrand, "Fair end-to-end window-based congestion control," IEEE/ACM Trans. on Networking, vol. 8, no. 5, pp. 556-567, October 2000.

[14] M. Mehyar, D. Spanos and S. H. Low, "Optimization Flow Control with Estimation Error," Proc. IEEE INFOCOM, March 2004.

[15] M. J. Osborne and A. Rubinstein, A Course in Game Theory. MIT Press, 1994.

[16] R. Srikant, The Mathematics of Internet Congestion Control. Birkhauser, 2004.

[17] D. M. Topkis, "Equilibrium points in nonzero-sum n-person submodular games," SIAM Journal of Control and Optimization, vol. 17, no. 6, pp. 773-787, November 1979.

[18] D. D. Yao, "S-modular games, with queueing applications," Queueing Systems, vol. 21, pp. 449-475, 1995.

[19] C. Yuen and P. Marbach, "Price-based rate control in random access networks," IEEE/ACM Trans. on Networking, vol. 13, no. 5, pp. 1027 1040, December 2005.

\section{APPENDIX: PROOFS}

\section{A. Theorem 1}

Proof: We first obtain the utility function of each link based on the update algorithm in (3). Assuming that there exists an equilibrium persistence probabilities $\mathbf{p}^{*}, \mathbf{p}^{\text {min }}<$ $\mathbf{p}^{*}<\mathbf{p}^{\max }$, then we see from (3) that $\mathbf{p}^{*}$ satisfies the following:

$$
\begin{aligned}
p_{l}^{*}= & p_{l}^{\max } p_{l}^{*} \prod_{n \in L_{t o}^{I}(l)}\left(1-p_{n}^{*}\right) \\
& +\beta_{l} p_{l}^{*} p_{l}^{*}\left(1-\prod_{n \in L_{t o}^{I}(l)}\left(1-p_{n}^{*}\right)\right)+p_{l}^{*}\left(1-p_{l}^{*}\right) .
\end{aligned}
$$

Since each link adjusts its own persistence probability to maximize its utility given persistence probabilities of the other link, from (14) and the first order necessary condition, each link $l$ has its utility function, $U_{l}(\mathbf{p})$, such that

$$
\begin{aligned}
\frac{\partial U_{l}(\mathbf{p})}{\partial p_{l}}= & p_{l}^{\max } p_{l} \prod_{n \in L_{t o}^{I}(l)}\left(1-p_{n}\right) \\
& +\beta_{l} p_{l} p_{l}\left(1-\prod_{n \in L_{t o}^{I}(l)}\left(1-p_{n}\right)\right) \\
& +p_{l}\left(1-p_{l}\right)-p_{l} .
\end{aligned}
$$

Hence, the utility function of link $l, U_{l}(\mathbf{p})$, which is unique up to a constant offset, is obtained as

$$
\begin{aligned}
U_{l}(\mathbf{p})= & \frac{1}{2} p_{l}^{\max } \prod_{n \in L_{t o}^{I}(l)}\left(1-p_{n}\right) p_{l}^{2} \\
& +\frac{1}{3} \beta_{l}\left(1-\prod_{n \in L_{t o}^{I}(l)}\left(1-p_{n}\right)\right) p_{l}^{3}-\frac{1}{3} p_{l}^{3} \\
= & p_{l}^{2} \prod_{n \in L_{t o}^{I}(l)}\left(1-p_{n}\right)\left(\frac{1}{2} p_{l}^{\max }-\frac{1}{3} p_{l}\right) \\
& -\frac{1}{3}\left(1-\beta_{l}\right) p_{l}^{3}\left(1-\prod_{n \in L_{t o}^{I}(l)}\left(1-p_{n}\right)\right) \\
= & R\left(p_{l}\right) S(\mathbf{p})-C\left(p_{l}\right) F(\mathbf{p}),
\end{aligned}
$$

where $R\left(p_{l}\right)=p_{l}\left(\frac{1}{2} p_{l}^{\max }-\frac{1}{3} p_{l}\right), C\left(p_{l}\right)=\frac{1}{3}\left(1-\beta_{l}\right) p_{l}^{2}$, $S(\mathbf{p})=p_{l} \prod_{n \in L_{t o}^{I}(l)}\left(1-p_{n}\right)$, and $F(\mathbf{p})=p_{l}(1-$ $\prod_{n \in L_{t o}^{I}(l)}\left(1-p_{n}\right)$.

It can be verified that utility function $U_{l}$ is quasi-concave in $p_{l}$. The action set $A_{l}=\left\{p_{l} \mid p_{l}^{\text {min }} \leq p_{l} \leq p_{l}^{\max }\right\}$ of each link $l$ is a nonempty compact convex subset of Euclidian space, and the utility function $U_{l}$ of each link $l$ is continuous and quasi-concave on $A_{l}$. Hence, by Proposition 20.3 in [15], there exists a Nash equilibrium.

Moreover, from (15), we can easily show that

$$
\frac{\partial U_{l}(\mathbf{p})}{\partial p_{l}}\left\{\begin{array}{l}
>0, \text { if } p_{l}<\frac{p_{l}^{\max } \prod_{n \in L_{t o}^{I}(l)}\left(1-p_{n}\right)}{1-\beta_{l}\left(1-\prod_{n \in L_{t o}^{I}(l)}\left(1-p_{n}\right)\right)} \\
<0, \text { otherwise }
\end{array}\right.
$$

Hence, we can characterize Nash equilibrium for persistence probabilities of links as

$$
p_{l}^{*}=\frac{p_{l}^{\max } \prod_{n \in L_{t o}^{I}(l)}\left(1-p_{n}^{*}\right)}{1-\beta_{l}\left(1-\prod_{n \in L_{t o}^{I}(l)}\left(1-p_{n}^{*}\right)\right)}, \forall l .
$$

\section{B. Theorem 4}

Proof: We have $\frac{\partial^{2} U_{l}(\mathbf{p})}{\partial p_{l} \partial p_{k}}$

$$
=\left\{\begin{array}{ll}
\prod_{\substack{n \in L_{t o}^{I}(l), n \neq k \\
0,}}\left(1-p_{n}\right)\left(\beta_{l} p_{l}^{2}-p_{l}^{\max } p_{l}\right), k \in L_{t o}^{I}(l) & \text { otherwise }
\end{array} .\right.
$$

Since $\beta_{l}<1$ and $p_{l} \leq p_{l}^{\max }$, the utility function is submodular ${ }^{4}$. Moreover, the action set of a link does not depend on the strategies of the other links. Hence, by applying Theorem 5.1 in [18], the proof is completed.

${ }^{4}$ If $U_{l}$ is twice differentiable and $\frac{\partial^{2} U_{l}(\mathbf{p})}{\partial p_{l} \partial p_{k}} \leq 0, \forall \mathbf{p} \in \times_{l \in L} A_{l} \forall k \neq l$, then $U_{l}$ is submodular. We refer readers to [17], [18] for more details on submodularity. 


\section{Lemma 1}

Proof: $\prod_{i \in L_{\text {to }}^{I}(l)}\left(1-p_{i}^{*}\right) \leq 1$. It is easy to check $p_{l}^{*}$ achieves its maximal $p^{\max }$ when $\prod_{i \in L_{t o}^{I}(l)}\left(1-p_{i}^{*}\right)=1$. Therefore $p_{l}^{*} \leq p^{\max }$.

If $p_{l}^{*}=0$, then $p_{i}^{*}=1$ for some $i \in L_{t o}^{I}(l)$. That is impossible as we know $p_{i}^{*} \leq p^{\max }<1$. Hence $p_{l}^{*}>0$.

If $p_{l}^{*}=p^{\max }$, then $\prod_{i \in L_{t o}^{I}(l)}\left(1-p_{i}^{*}\right)=1$. That is again impossible as $p_{i}^{*}>0$. Hence $p_{l}^{*}<p^{\max }$.

\section{Theorem 5}

Proof: The best response updating rule is defined as following:

$$
p_{l}(t+1)=p^{\max } \frac{\prod_{i \in L_{t o}^{I}(l)}\left(1-p_{i}(t)\right)}{1-\beta\left(1-\prod_{i \in L_{t o}^{I}(l)}\left(1-p_{i}(t)\right)\right)} .
$$

Its equilibrium is characterized by (11). We now set up uniqueness and convergence together by showing (18) is a contraction mapping. We first cite the following basic theorem [1] that we will use.

Contraction Mapping Theorem. Let $M$ be a complete metric space and $f: M \rightarrow M$ a mapping. Assume there is a constant $k$, where $0 \leq k<1$, such that $d(f(u), f(v)) \leq k d(u, v)$, for all $u, v \in M$; such an $f$ is called a contraction. Then $f$ has a unique fixed point; that is, there exists a unique $u^{*} \in M$. Furthermore, the sequence $u(t+1)=f(u(t))$ converges to the unique fixed point.

Let $M$ be the Euclidean space and consider any vector norm. Let $d($.$) be the induced distance function by the vector$ norm. We have

$$
\begin{aligned}
d(f(u), f(v)) & =\|f(u)-f(v)\| \\
& \leq\left\|\frac{\partial f}{\partial x}\right\|\|(u-v)\| \\
& =\left\|\frac{\partial f}{\partial x}\right\| d(u, v) .
\end{aligned}
$$

The matrix norm used here is induced by the vector norm too. The inequality follows from the property of matrix norm. Hence it is clear that if we have the Jocobian $\left\|\frac{\partial f}{\partial x}\right\|<1-\epsilon$ everywhere for some positive $\epsilon$, we can let $k=1-\epsilon<1$ and the Contraction Mapping Theorem applies. ${ }^{5}$

We now derive conditions using $\|.\|_{\infty}$ for (18) to be a contraction map. Its Jacobian $\mathbf{J}$ is defined by

$$
J_{l j}=\frac{\partial p_{l}(t+1)}{\partial p_{j}(t)} .
$$

It is straightforward to check

$$
J_{l j}= \begin{cases}0, & j \notin L_{t o}^{I}(l) \\ -p^{\max } \frac{(1-\beta) \prod_{i \in L_{t o}^{I}(l), i \neq j}\left(1-p_{i}\right)}{\left(1-\beta\left(1-\prod_{i \in L_{t o}^{I}(l)}\left(1-p_{i}\right)\right)\right)^{2}}, & j \in L_{t o}^{I}(l)\end{cases}
$$

It then follows that

$$
\|\mathbf{J}\|_{\infty}=\max _{l}\left\{p^{\max } \sum_{j \in L_{t o}^{I}(l)} \frac{(1-\beta) \prod_{i \in L_{t o}^{I}(l), i \neq j}\left(1-p_{i}\right)}{\left(1-\beta\left(1-\prod_{i \in L_{t o}^{I}(l)}\left(1-p_{i}\right)\right)\right)^{2}}\right\} .
$$

${ }^{5}$ As $\epsilon$ can be arbitrarily small, the later derivation will use 1 instead.
For any $j \in L_{t o}^{I}(l)$, define

$$
\pi(l, j)=\prod_{i \in L_{t o}^{I}(l), i \neq j}\left(1-p_{i}\right)
$$

and

$$
M(l, j)=\frac{(1-\beta) \prod_{i \in L_{t o}^{I}(l), i \neq j}\left(1-p_{i}\right)}{\left(1-\beta\left(1-\left(1-p_{j}\right) \prod_{i \in L_{t o}^{I}(l), i \neq j}\left(1-p_{i}\right)\right)\right)^{2}} .
$$

We have

$$
M(l, j)=\frac{(1-\beta) \pi(l, j)}{\left(1-\beta\left(1-\left(1-p_{j}\right) \pi(l, j)\right)\right)^{2}}
$$

and

$$
\frac{d M(l, j)}{d \pi(l, j)}=\frac{(1-\beta)\left(1-\beta-\beta \pi(l, j)\left(1-p_{j}\right)\right)}{\left(1-\beta\left(1-\left(1-p_{j}\right) \pi(l, j)\right)\right)^{3}} .
$$

It then follows that, if $\frac{1-\beta}{\beta\left(1-p_{j}\right)} \leq 1, M(l, j)$ achieves its maximum value of $\frac{1}{4 \beta\left(1-p_{j}\right)}$ when $\pi(l, j)=\frac{1-\beta}{\beta\left(1-p_{j}\right)}$, i.e., $\prod_{i \in L_{t o}^{I}(l)}\left(1-p_{i}\right)=\frac{1-\beta}{\beta}$. If $\frac{1-\beta}{\beta\left(1-p_{j}\right)} \geq 1, M(l, j)$ reaches its maximum value of $\frac{1-\beta}{\left(1-\beta+\beta\left(1-p_{j}\right)\right)^{2}}$ when $\pi(l, j)=1$. $^{6}$ Therefore, we conclude that

$$
\begin{aligned}
\|\mathbf{J}\|_{\infty} & =\max _{l}\left\{p^{\max } \sum_{j \in L_{t o}^{I}(l)} M(l, j)\right\} \\
& \leq \max _{l}\left\{\frac{p^{\max }\left|L_{t o}^{I}(l)\right|}{4 \beta\left(1-p^{\max }\right)}\right\} \\
& \leq \frac{p^{\max } K}{4 \beta\left(1-p^{\max }\right)} .
\end{aligned}
$$

By assumption in the theorem, we conclude $\|J\|_{\infty}<1$. Hence, (18) is a contraction mapping and both uniqueness and global convergence are guaranteed [1].

\section{E. Derivation of the upperlimit}

We now show the upperbound of $p^{\max }$ cannot be made independent of $L$ via above method, by deriving an upperlimit considering the system in which each link interfere each other (i.e., $\left.L_{t o}^{I}(l)=E-\{l\}, \forall l\right)$ that takes into account the relation among $M(l, j)$ for different $j$, which has been neglected in previous derivation. We have

$$
\|\mathbf{J}\|_{\infty}=p^{\max } \sum_{j \neq l} \frac{(1-\beta) \prod_{i \neq l, j}\left(1-p_{i}\right)}{\left(1-\beta\left(1-\prod_{i \neq l}\left(1-p_{i}\right)\right)\right)^{2}} .
$$

Let $y_{i}=1-p_{i}$, then

$$
\|\mathbf{J}\|_{\infty}=p^{\max }(1-\beta) \sum_{j \neq l} \frac{\prod_{i \neq l, j} y_{i}}{\left(1-\beta\left(1-\prod_{i \neq l} y_{i}\right)\right)^{2}} .
$$

We are interested in finding its maximum with constraint $y_{i} \in$ $\left[1-p^{\max }, 1\right]$, it is at least as big as the the maximum of $V(y)=p^{\max }(1-\beta) \frac{(L-1) y^{L-2}}{\left(1-\beta+\beta y^{L-1}\right)^{2}}$, where $y \in\left[1-p^{\max }, 1\right]$. ${ }^{6} \mathrm{It}$ is interesting to note that for standard parameter setting $\beta=0.5$, $\frac{1-\beta}{\beta\left(1-p_{j}\right)} \geq 1$ always holds. 


$$
\begin{aligned}
\frac{d V(y)}{d y}= & p^{\max }(1-\beta) \frac{\left(1-\beta+\beta y^{L-1}\right)(L-1)(L-2) y^{L-3}}{\left(1-\beta+\beta y^{L-1}\right)^{3}} \\
& -\frac{2 \beta(L-1)^{2} y^{2 L-4}}{\left(1-\beta+\beta y^{L-1}\right)^{3}}
\end{aligned}
$$

Solving the optimality condition $\frac{d V(y)}{d y}=0$ gives the critical value

$$
y^{c}=\left(\frac{(1-\beta)(L-2)}{\beta L}\right)^{\frac{1}{L-1}} .
$$

Therefore, if $y^{c}<1-p^{\max }, \max (V(y))=V\left(1-p^{\max }\right)$; if $y^{c}>1,{ }^{7} \max (V(y))=V(1)$; otherwise, $\max (V(y))=$ $V\left(y^{c}\right)$. Imposing $\max (V(y))<1$, we achieve the limit for upperbound for $p^{\max }$ via using contraction mapping and infinity norm.

Notice that to derive various upperbounds on $p_{l}^{\max }$ allowed, we bound $\max \{\|\mathbf{J}\|\}<M$ and then impose conditions for $M<1$, whereas to derive an upperlimit on how good such upperbounds can be, we find $\max \{\|\mathbf{J}\|\}>M^{\prime}$ and then impose conditions for $M^{\prime}<1$.

\section{F. Theorem 6}

Proof: The proof relies on Theorem 6.2 in [4], a variation of which is stated below.

Stochastic Subgradient Convergence Theorem. Consider the maximization of a concave continuous one-dimensional function $F(x)$ in $x \in[a, b]$, and let $X^{*}$ be a set of optimal solutions. Consider the following stochastic subgradient projection method:

$$
\begin{gathered}
x(t+1)=\max \{a, \min \{b, x(t)+s(t) \xi(t)\}\}, t=0,1, \ldots \quad(23) \\
F\left(x^{*}\right)-F(x(t)) \leq E\{\xi(t) \mid x(0), \cdots, x(t)\}\left(x^{*}-x(t)\right)+\gamma_{0}(t),
\end{gathered}
$$

where $\gamma_{0}(t)$ may depend on $(x(0), \cdots, x(t)), x^{*} \in X^{*}$, and $s(t)$ is the step size that satisfies

$s(t) \geq 0, \sum_{t=0}^{\infty} s(t)=\infty, \sum_{t=0}^{\infty} E\left\{s(t)\left|\gamma_{0}(t)\right|+s^{2}(t)|\xi(t)|^{2}\right\}<\infty$.

Then $\lim x(t) \in X^{*}$ with probability 1 .

For our proof, we map the elements of the updates in (13) into the elements of the algorithm in $(23,24,25)$. Define

$$
p_{l}^{\text {reflex }}=p^{\max } \frac{\Pi_{n \in L_{t o}^{I}(l)}\left(1-p_{n}\right)}{2\left(1-\beta\left(1-\Pi_{n \in L_{t o}^{I}(l)}\left(1-p_{n}\right)\right)\right)} .
$$

It can be verified that $U_{l}\left(p_{l}\right)$ is strictly concave in $p_{l} \in$ $\left[p_{l}^{\text {reflex }}, p^{\max }\right]$ for fixed $p_{-l}$. Also the unique maximizer of $U_{l}\left(p_{l}\right)$ is $p_{l}^{B R}=2 p_{l}^{\text {reflex }}$. It can be further shown that under conditions 2 and 3 in the theorem, $\tilde{p}_{l}^{\text {min }}$ satisfies

$$
\max \left\{p^{\min }, p_{l}^{\text {reflex }}\right\} \leq \tilde{p}_{l}^{\min } \leq p_{l}^{B R} \leq p^{\max }
$$

for any feasible value $\left.p_{n}, n \in L_{t o}^{I}(l)\right)$. These enable us to establish the following mappings: $p_{l} \rightarrow x, U_{l}\left(p_{l}\right) \rightarrow F(x)$,

\footnotetext{
${ }^{7}$ This can only happen if $\beta<\frac{L-2}{2 L-2}$, and cannot happen with $\beta=0.5$.
}

$\left[\tilde{p}_{l}^{\min }, p^{\max }\right] \rightarrow[a, b]$ and $\left\{p_{l}^{B R}\right\} \rightarrow X^{*}$. Here $p_{l}^{B R}$ is the best response solution as in (10).

Now we map $v_{l}(t)$ into $\xi(t)$. Since

$E\left\{v_{l}(t) \mid p_{l}(0), \cdots, p_{l}(t)\right\}=E\left\{v_{l}(t) \mid p_{l}(t)\right\}=\left.\frac{\partial U_{l}\left(p_{l}\right)}{\partial p_{l}}\right|_{p_{l}=p_{l}(t)}$,

inequality (24) is satisfied with $\gamma_{0}(t)=0$. Finally, (25) is satisfied under condition 1 and the fact that $\left|v_{l}(t)\right|^{2} \leq$ $\left(p^{\max }\right)^{2}$. All the conditions of the Stochastic Subgradient Convergence Theorem are satisfied, and $p_{l}(t)$ converges to the best response solution with probability 1 . 